\title{
Construction and Characterization of a Replication-Deficient Adenovirus Expressing Rat-Soluble Interleukin-6 Receptor
}

\author{
Vincent Thibault, ${ }^{* \dagger}$ Bernard Terlain, ${ }^{\dagger}$ Frank L. Graham, ${ }^{\neq}$ \\ and Jack Gauldie* \\ ${ }^{*}$ Molecular Virology and Immunology Program, Department of \\ Pathology and ${ }^{\ddagger}$ Biology, McMaster University, Hamilton, \\ Ontario, Canada \\ ${ }^{\dagger}$ Laboratoire de Pharmacologie et URA CNRS 1288, Faculté de \\ Médecine, Vandoeuvre les Nancy, France
}

\begin{abstract}
Background: The pleiotropic cytokine interleukin-6 mediates its multiple effects at the cell level through a multimeric receptor consisting of a binding protein (gp80) and a signal transducer (gp130). A soluble form of gp80 (sIL-6R or gp55) is found released from the surface of cells and appears to possess interleukin-6 (IL-6) agonist activity. Increases in circulating levels of sIL-6R have been reported in different pathological conditions but the precise role of this protein in vivo remains unknown.

Materials and Methods: The cDNA encoding the extracellular domain of the rat IL-6R (sIL-6R) with an appropriate leader sequence has been cloned into the $\mathrm{El}$ region of an adenovirus vector under the control of the hCMV promoter (Ad5.sIL-6R).

Results: Infection of different human or rodent cell lines with Ad5.sIL-6R leads to extended production of recom-
\end{abstract}

\begin{abstract}
binant sIL-6R protein into the culture media. The kinetics of transgene expression depends both on the cell type and the species. sIL-6R produced in this manner is biologically active as it confers responsiveness of human hepatoma cells (HepG2) to rat IL-6 stimulation. Adenovirus vectors have been shown to be highly effective for transient delivery of cytokines in vivo. Antibodies against recombinant rat soluble $\mathrm{IL}-6 \mathrm{R}$ were generated and an ELISA developed that allowed us to quantify sIL-6R concentrations. The sIL-6R expressing adenovirus vector has been instilled intratracheally into rats and induced an increase in lung sIL-6R concentration from Day 1 up to Day 10. We demonstrate the potency of our system to deliver in vivo or in vitro soluble cytokine receptors in a prolonged but transient manner.
\end{abstract}

\section{INTRODUCTION}

Infection or trauma inflicted on an organism is followed by an immediate local and systemic response aimed at halting tissue destruction, removing the infecting organisms or tissue, and restoring the physiologic balance. One of the most potent mechanisms involved in this reac-

Address correspondence and reprint requests to: Dr. J. Gauldie, Department of Pathology, McMaster University, 1200 Main Street West, Hamilton, Ontario L8N 3Z5, Canada. Phone: 905-521-2100, ext. 6332; fax: 905-577-0198; e-mail: Gauldie@FHS.McMaster.CA tion is the release of cytokines into the circulation in an ordered and precise manner, with interleukin-6 (IL-6) in combination with others playing a very important role during such acute pathologic conditions. IL- 6 has been shown more specifically to regulate the acute phase reaction, the immune response, and many other crucial components of the homeostatic response $(1,2)$. IL-6 acts at the surface of cells through a specific receptor, gp80 or $\alpha$ chain, which binds the IL- 6 ligand with low affinity. After the formation of this IL-6/IL-6R complex, a third component, gpl30 or $\beta$ chain, is necessary to transduce the signal to the cell. This last step involves 
formation of a high-affinity interaction between IL- 6 and the chains of the receptor. Recent data seem to indicate formation of a hexameric complex of two molecules each of IL- $6, \alpha$ chain, and $\beta$ chain $(3,4)$.

The short intracytoplasmic domain of gp80 has been shown not to be needed to trigger a cellular IL-6-mediated response. Indeed, a recombinant protein consisting of the extracellular domain of gp80, gp55-soluble IL-6R (sIL-6R), is still able to bind IL-6, and this complex, IL-6/sIL$6 \mathrm{R}$, by interacting with gp 130 , induces an intracellular signal $(5,6)$. Although we possess much information on the possible roles of sIL-6R in vitro, its precise role in vivo remains unclear. An effect on the central nervous system has been suggested (7-13). Availability of immunoassays to measure sIL-6R reveals the relatively high circulating concentrations of this protein either under normal conditions or during pathologic states (14-16). More recently, severe sepsis and fatal outcome have been associated with elevated levels of sIL-6R (17).

Methods to investigate the physiologic role of a protein depend often on availability of the molecule under consideration in recombinant purified form. In addition to injecting recombinant material to an animal, a novel approach to induce a transient increase in the level of a defined protein is to generate a vector carrying the gene encoding for it. Recent data from our lab and others have proven the potency of recombinant adenoviruses to deliver cytokine genes to various tissues and thereby induce sustained increases in circulating levels of particular cytokines $(18,26,39-41)$.

To investigate the physiologic role of sIL-6R in an animal model, we constructed a replication-deficient adenovirus vector carrying the gene encoding the extracellular domain of IL-6R (Ad5.rsIL-6R). We describe the molecular strategy we used as well as the characterization of the protein synthesized by this recombinant virus in different cell lines. As previously shown with other adenoviruses expressing cytokines, Ad5.rsIL-6R-infected cells secrete high amounts of sIL-6R in vitro over a period of at least 10 days. The recombinant protein is biologically active, and intratracheal administration of the recombinant vector to rats induces the production of sIL-6R within lung tissue over a period of at least 10 days, as detected in bronchoalveolar lavage (BAL) fluid.

\section{MATERIALS AND METHODS Generation of cDNA Encoding Rat-Soluble IL-6R (p55)}

Total RNA was prepared from primary rat hepatocytes as previously described (19). A reverse transcription reaction was conducted according to manufacturer protocol using poly-dT as primer and M-MLV reverse transcriptase (GIBCO BRL, Gaithersburg, MD). The cDNA obtained was amplified by polymerase chain reaction using a set of primers (Mobix, McMaster University) and Vent DNA polymerase (New England Biolabs, Beverly, MA). The first primer spanned the $5^{\prime}$ end of the region encoding the signal peptide of rat IL-6R (according to Baumann et al. [20]) region [GGGCTCGAGGTAG AAGGAACCATG] and the second spanned the junction between the extracellular domain and the transmembrane domain of rat IL-6R [AG GCGGAATTCATTGCATGGGGC] (the Xhol site and the EcoRI/Stop codon are underlined). The cDNA obtained was cloned into the Xhol/EcoRI sites of Bluescript plasmid (Stratagene, La Jolla, CA) to give "rsIL-6R Blue" and sequenced.

\section{Adenovirus Vector}

The cDNA encoding rat sIL-6R (1069 bp) was cut from rsIL-6R Blue by restriction digest with EcoRI/Kpnl and subcloned into the multicloning site of pACCMV.PLPASR (+) (kindly provided by Dr. Bob Gerrard, Department of Cardiology, Howard Hughes Medical Institute, University of Texas, Dallas, TX). This plasmid contains sequentially the left end (1.3 maps unit [mu]) of the adenovirus type 5 (Ad5) genome, the hCMV early promoter $(760 \mathrm{bp})$, the pUC 19 multicloning site, the Simian Virus 40 splicing and polyadenylation signal, and map units 9.3 to 17 of Ad5 genome (45). pACCMV.rsIL-6R was characterized by restriction digest mapping and then cotransfected with pBHG10 plasmid into 293 cells by calcium phosphate precipitation. pBHG 10 lacks the packaging signal $(\Delta \psi 194-358$ bp) and cannot generate infectious viral vector unless recombined with a second plasmid containing the left end of the genome. Recombinant viruses obtained were plaque purified and their DNA characterized by HindIII restriction digest mapping. One clone, Ad5.rsIL-6R, was further grown in spinner culture of 293 N3S cells. Purification of the high-titer virus stock was done by cesium chloride gradient centrifugation and PD-10 gel filtration column as previously de- 
scribed (22). The titer (plaque-forming units/ml) of stock virus preparations was determined by infection of 293 cells and stored at $-80^{\circ} \mathrm{C}$ until use. Owing to the deletion in the $\mathrm{El}$ region, recombinant viruses obtained by recombination of pBHGl0 and pACCMV.rsIL-6R are replication-deficient. Control virus was Ad5.d170-3 (E1-deleted empty vector) or Ad5.E1.LacZ (LacZ insert in El).

DNA and RNA were obtained from 293 cells as previously described and run on Southern and Northern blot according to previously described protocols (22). The rat sIL-6R probe used was the Styl fragment corresponding to the extracellular domain (63-931 bp).

\section{Animals}

Sprague-Dawley rats were injected intratracheally as previously described (21). In brief, rats were anesthesized, incised at the trachea level, and $300 \mu \mathrm{l}$ of virus diluted to the proper concentration in sterile PBS were slowly instilled with a 21-G needle. At different time points, rats were euthanized and the lungs lavaged (BAL) with 3 $\mathrm{ml}$ of sterile PBS.

\section{Production of rsIL-6R in vitro}

Primary human lung (Human LF) and rat lung fibroblast (Rat LF) lines and HepG2 human hepatoma or $\mathrm{H} 35$ rat hepatoma cells were grown to subconfluency in 24-well plates in F11 medium, $5 \%$ fetal calf serum (FCS), $1 \%$ penicillin/streptomycin (GIBCO) and infected with recombinant Ad5 vectors for $1 \mathrm{hr}\left(37^{\circ} \mathrm{C}\right)$ at a multiplicity of infection (MOI) of 25 to 100. Medium was removed, cells were washed once with culture medium and further incubated with fresh medium $(500 \mu \mathrm{l})$ containing 1\% FCS. Every $24 \mathrm{hr}$ the medium was collected and replaced by new medium.

\section{Infection and Stimulation of HepG2 Cells and Determination of Acute Phase Proteins}

HepG2 human hepatoma cells were grown in 24-well plates to subconfluency, medium (aMEM, $10 \%$ FCS, $1 \%$ penicillin/streptomycin; GIBCO) was removed, and the cells were infected with Ad5.rsIL-6R or Ad5.d170-3 (control) at a MOI of 100 for $1 \mathrm{hr}$ at $37^{\circ} \mathrm{C}$. The medium was removed and stimulatory cytokines or diluent alone were added in $300 \mu \mathrm{l}$ of fresh me- dium containing $2 \%$ FCS. After 3 days of stimulation, supernatant was collected and analyzed by immunorockets in agarose gel using anti alpha-1 antichymotrypsin (AHPO3; Serotec, Bicester, U.K.) as previously described (37).

\section{Antibody to Rat SIL-6R}

Specific antibody to rat sIL-6R was generated by initial immunization of rabbits with $10^{9}$ plaqueforming units (pfu) of recombinant adenovirus expressing rat sIL-6R. One month later, rabbits were boosted with $20 \mu \mathrm{g}$ of purified rat sIL-6R derived from a baculovirus expression system as previously described (42) and repeated at monthly intervals. Rabbits were bled 7 days after each injection and each serum tested by Western blot for the ability to detect recombinant protein expressed in different systems $(E$. coli, baculovirus, adenovirus). IgG from the most immunoreactive antiserum and control nonimmune serum was purified on a protein A sepharose column (Pharmacia, Gaithersburg, MD) according to the manufacturer's protocol.

\section{Western Blot}

Proteins were run on a $7.5 \%$ or $10 \%$ polyacrylamide gel under reducing conditions. The gels were transferred onto a nitrocellulose membrane (Immobilon). After blocking for $1 \mathrm{hr}$ with $5 \%$ blotto, $1 \%$ BSA, Tween $1 \%$ PBS, the antibodies were added to a dilution of 1:5,000 in the same solution. Development was done with a secondary antibody (goat anti-rabbit; Sigma, St. Louis, MO) labeled with peroxidase and a chemiluminescence system (Dupont-NEN, Boston, MA) according to manufacturer's specification.

\section{ELISA}

Samples to be measured for concentration of rat sIL-6R were coated on Linbro (Flow Laboratories, McLean, VA) ELISA plate overnight at $4^{\circ} \mathrm{C}$. When dilutions were necessary, phosphate-buffered saline (PBS) was used. A preparation containing a known amount of recombinant rat sIL-6R purified from a baculovirus expression system was used as a standard. Concentration range of the standard curve extended from 4 to $500 \mathrm{ng} / \mathrm{ml}$. All samples, dilutions, and standards were run in duplicate and the mean of two wells was calculated for interpretation. Briefly, $100 \mu \mathrm{l}$ of the samples were coated overnight at $4^{\circ} \mathrm{C}$, the plate was washed three times with Tween-PBS 


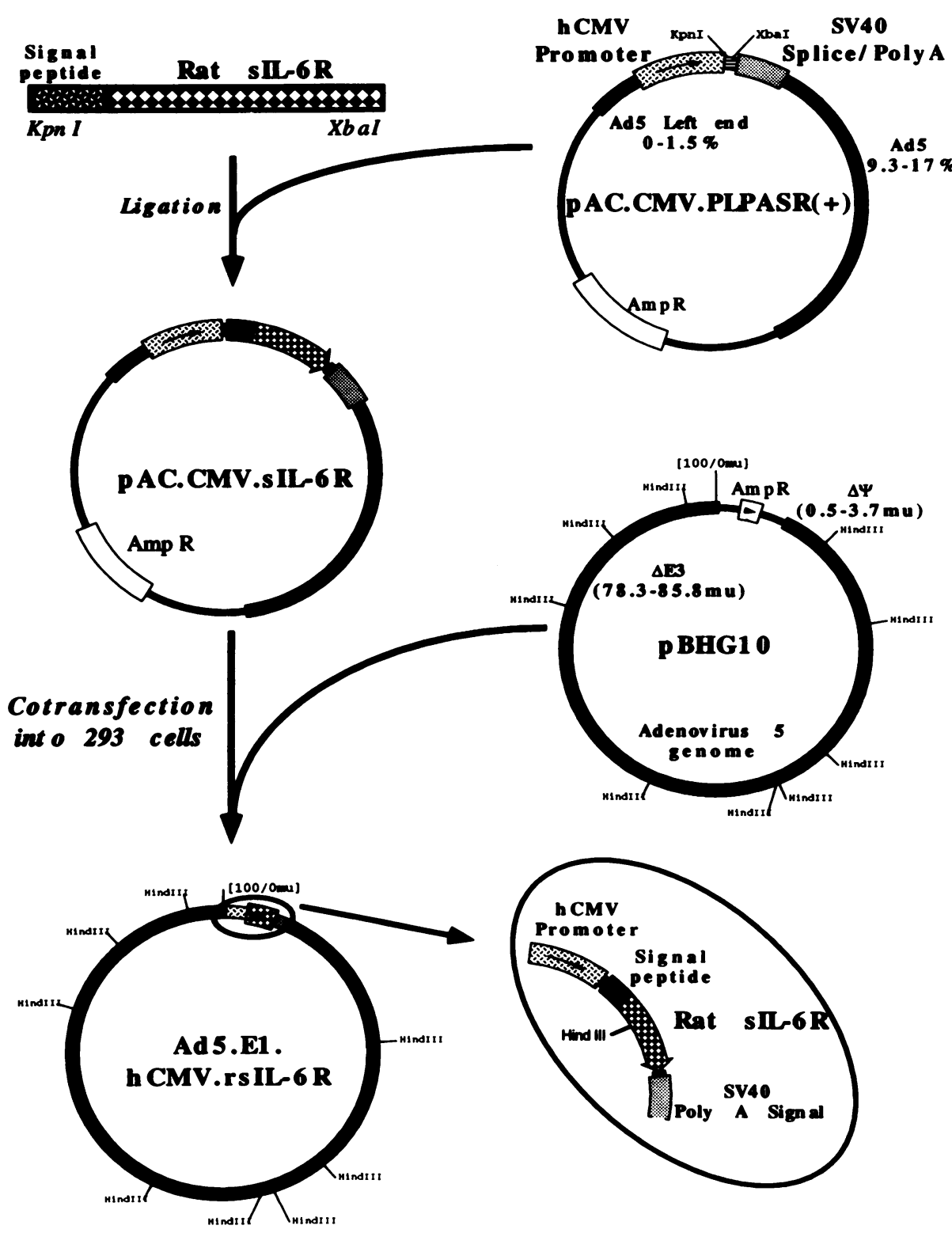

FIG. 1. Summary of recombinant adenovirus construction The cDNA encoding the extracellular (soluble) domain of rat interleukin-6 receptor was cloned into plasmid pACCMV to give pACCMV.rsIL-6R that was cotransfected with pBHG10 into 293 cells.
$(0.05 \%)$ at room temperature and blocked with $100 \mu \mathrm{l}$ of $1 \%$ gelatin in T-PBS for $1 \mathrm{hr}$ at $37^{\circ} \mathrm{C}$ in a humid atmosphere, then washed three times as previously. One hundred microliters of specific antibody diluted 1:1000 was added and left for 1 hr at $37^{\circ} \mathrm{C}$. After three washes, a secondary antibody consisting of goat anti-rabbit labeled with peroxidase diluted 1:500 (Sigma) was added, and after $45 \mathrm{~min}$ the plate was washed three times and the enzyme substrate was added (ABTS, Sigma). The plate was read on a spectrophotometer at $492 \mathrm{~nm}$ against a blank consisting of a well coated with PBS only. The data were analysed using the software Deltasoft II (BioMetallics). When BAL samples were analyzed, several dilutions were done (from $1: 10$ to $1: 1280$ ) in dupli- cate and only the dilutions falling within the standard range were used for interpretation.

\section{RESULTS}

\section{Characterization of Recombinant} Adenovirus Expressing Rat sIL-6R

The recombinant virus encoding rat sIL-6R, Ad5.rsIL-6R, was constructed according to the protocol summarized in Fig. 1. A viral plaque was isolated after double plaque isolation and amplified on 293 cells according to previously described techniques (22). After appearance of full cytopathic effect (CPE), cells were harvested 

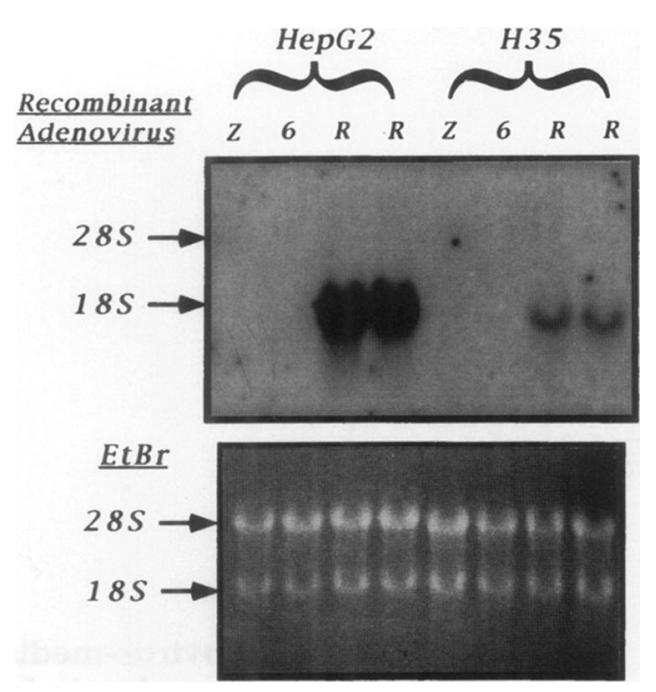

FIG. 2. Ad5.rsIL-6R cell infection gives rise to specific mRNA for rat SIL-6R

Total RNA from different recombinant adenovirusinfected HepG2 or H35 cells were analyzed by Northern blot and probed with an sIL-6R-specific

${ }^{32} \mathrm{P}$-labeled oligonucleotide. The upper panel shows the specific hybridization only with RNA extracted from Ad5.rsIL-6R-infected cells (lanes R), whereas Ad5.E1.LacZ (lanes Z) or Ad5.IL-6 (vector expressing rat IL-6) (lanes 6) do not give any signal. The bottom panel is the gel stained with ethidium bromide.

and DNA extracted. Digestion of viral DNA with HindIII and electrophoresis onto an agarose gel stained with ethidium bromide showed the expected pattern. Specific bands were hybridized with a probe for sIL-6R on a Southern blot from the same gel (data not shown).

To characterize the production of proper transcripts from cells infected with Ad5.sIL-6R, we infected human (HepG2) or rat (H35) hepatoma cells with different recombinant viruses at a MOI of 100 . The cells were collected 3 days after infection and RNA was analyzed by Northern blot hybridization using a specific probe for the extracellular domain of sIL-6R. As shown in Fig. 2, we could detect specific mRNA for sIL-6R only in the lanes corresponding to cells infected with Ad5.rsIL-6R.

\section{Cell Infection with Ad5.rsIL-6R Induces Production of Rat sIL-6R}

Three different cell lines, HepG2, H35, or a primary human lung cell line (Human LF) were infected with either Ad5.rsIL-6R or Ad5.d170-3 as control at a MOI of 100 . Crude supernatants

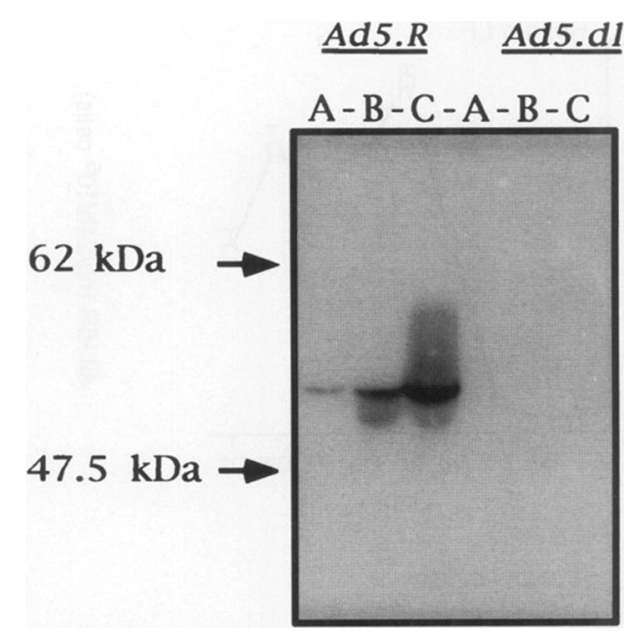

FIG. 3. Antibodies generated in rabbits recognize specifically rat sIL-6R

H35 (lanes A), HepG2 (lanes B), or human lung fibroblasts (lanes $C$ ) were infected with either Ad5.rsIL-6R or Ad5.d170-3, and supernatants of day 5 were run on $8.5 \%$ SDS-PAGE. After transfer, the Western blot membrane was probed with specific rat sIL-6R antibodies and revealed by a chemiluminescent reaction.

were collected 5 days after infection, centrifuged, and run on a Western blot. Proteins were revealed with the primary antibody directed against rat sIL-6R. Figure 3 shows clearly a specific band migrating around $55 \mathrm{kD}$, indicating the presence of sIL-6R only in the supernatants of cells infected with Ad5.sIL-6R and not in those infected with the control virus.

\section{Kinetics of Production of Rat sIL-6R in Rodent and Human Cells Infected with Ad5.rsIL-6R}

Since adenoviruses do not integrate into the genome of host cells and stay as an episomal form of DNA, it is important to define the kinetics of protein expression, especially the maximal time of recombinant protein production. Rat or human lung fibroblasts and hepatoma cells were infected with Ad5.rsIL-6R at a MOI of 10 at Day 0 and the medium was collected and replaced every day for 9 days. Depending on the cell line and the species, the kinetics of production were quite different (Fig. 4). With lung fibroblasts we could detect rsIL-6R protein from Day 3 up to Day 9 with a peak of expression around Day 5; with hepatoma cells, protein expression occurred earlier in time and stayed at a constant level for 

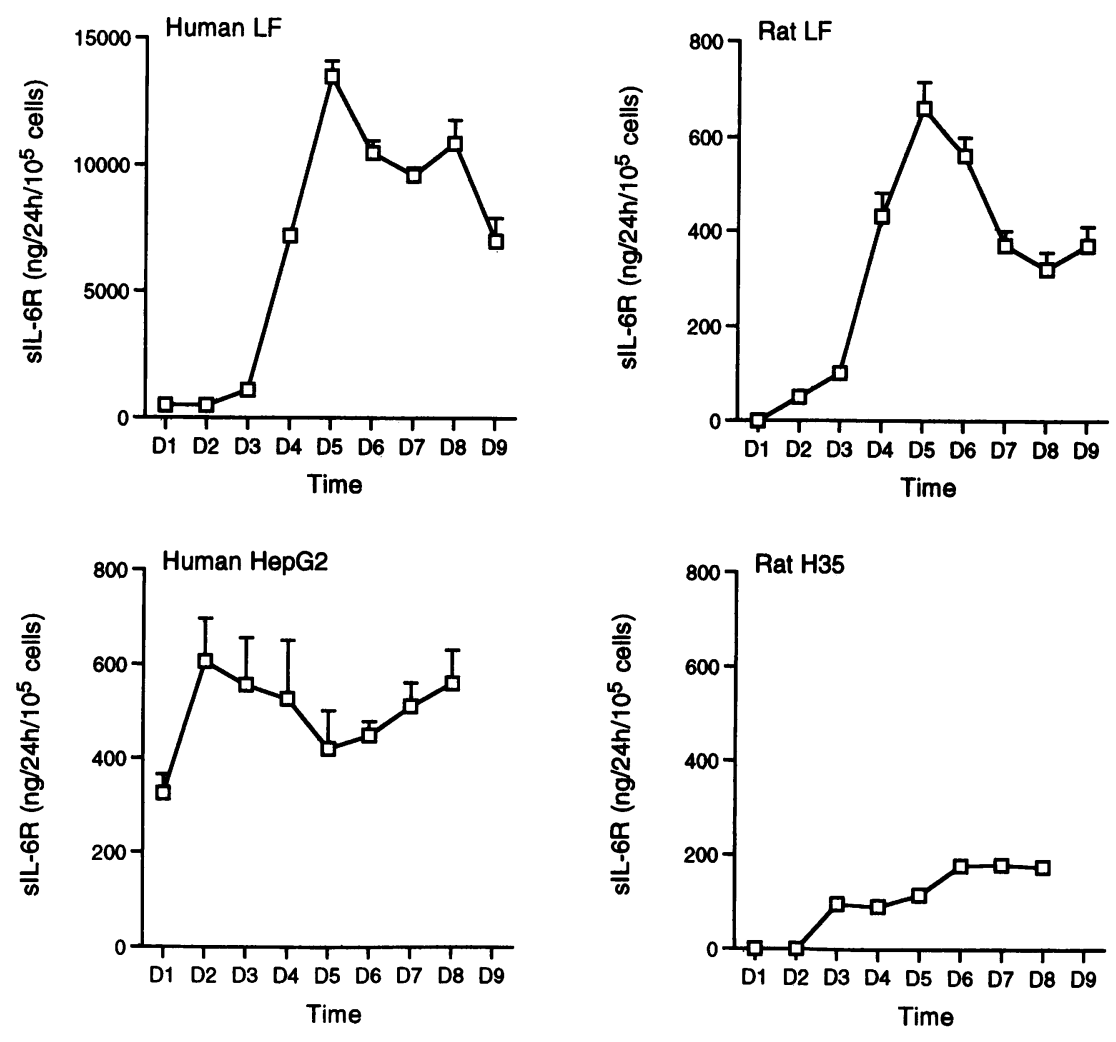

FIG. 4. Adenovirus-mediated rat sIL-6R expression in four different cell lines

Human lung fibroblast (Human LF), rat lung fibroblast (Rat LF), human HepG2, or rat H35 hepatoma cell lines were infected with Ad5.rsIL-6R and supernatants were collected every $24 \mathrm{hr}$ for 9 days and subjected to a specific ELISA for rat sIL-6R. Results are expressed as mean \pm SEM.

5 more days. In both cases the time-limiting factor appeared to be cell survival.

\section{Rat sIL-6R Produced from Ad5.rsIL-6R- Infected Cells Is Biologically Active}

IL-6 and its receptor are fairly conserved among species but generally, rodent IL- 6 cannot act on human cells, whereas human IL- 6 acts well on rodent cells. As we have previously shown, rat IL-6 can stimulate HepG2 cells to secrete human acute-phase proteins, only with the concomitant presence of recombinant rat sIL-6R (42). This is the result of the interaction of rat IL- 6 with rat sIL-6R, forming a complex which is then able to interact with human gp130 and trigger the intracellular signal.

Human hepatoma cells (HepG2) were left noninfected or infected with either Ad5.sIL-6R or Ad5.EI.LacZ as a control at a MOI of 10. One day after infection, at a point where rat sIL-6R is being secreted from the cells (see Fig. 4), the medium was changed and replaced with fresh medium containing different cytokines alone or in combination. The cells were stimulated for 2 days and the supernatants collected for determination of human acute-phase protein concentra- tions. Although rat IL-6 was not able to induce HepG2 cell stimulation per se, Ad5.rsIL-6R-infected cells responded to rat IL- 6 stimulation to the same extent as those stimulated by human IL-6 (Fig. 5). The cells infected by the control virus (Ad5.E1.LacZ) did not respond to rat IL-6 stimulation.

\section{In Vivo Production of Rat sIL-6R after Intratracheal Injection of Ad5.rsIL-6R}

Rats were injected intratracheally with $10^{9} \mathrm{pfu}$ of Ad5.rsIL-6R or control virus. Bronchoalveolar lavage (BAL) was performed and ELISA data showed kinetics of protein production to be very similar to what we saw in vitro: as early as Day 1 , we were able to detect the presence of recombinant rsIL-6 $R$ with concentrations of protein peaking at Day 2 and slowly decreasing to below the limit of detection after Day 10 in the Ad5.rsIL-6R-treated animals. None of the BAL samples recovered from rats injected with the control virus showed detectable concentrations of rat sIL-6R. BAL samples were subjected to Western blot analysis and characteristic bands at 50-55 kD were readily detected in Day 2 to 7 lavage fluids (Fig. 6). 


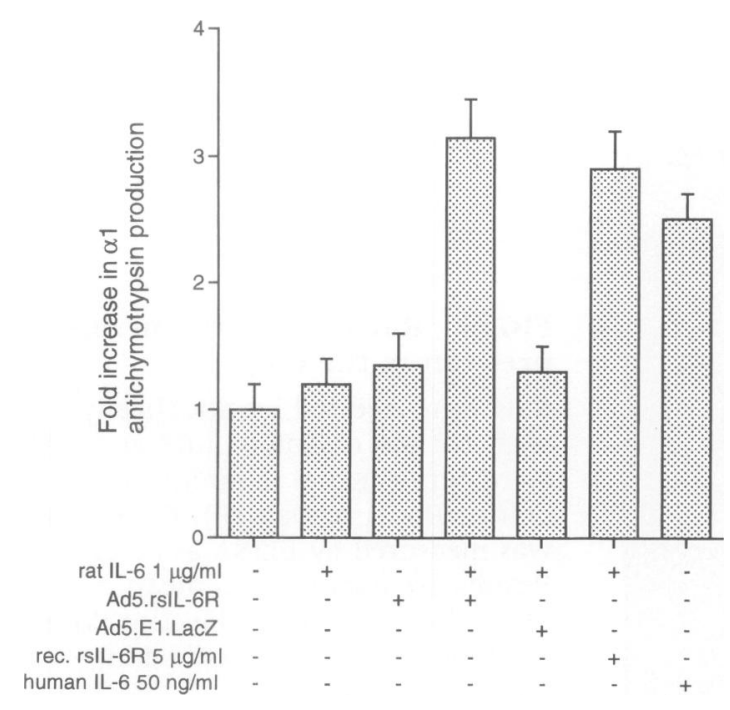

FIG. 5. Adenovirus-mediated rat sIL-6R expression confers HepG2 responsiveness to rat IL-6

Two-day supernatants from adenovirus-infected HepG2 cells were analyzed for $\alpha 1$ anti-chymotrypsin content by immunoelectrophoresis. The graph shows the relative amount of protein produced under different stimulation conditions with rat IL-6. Stimulation by human IL- 6 is shown as control. Results are expressed as mean \pm SEM.

\section{DISCUSSION}

The soluble extracellular domain of the gp80 IL-6 receptor (sIL-6R), a 45-55 kD glycoprotein, has been detected at significant levels in biologic fluids such as urine, cerebrospinal fluids, and synovial fluids $(17,43,44)$. However, despite evidence in vitro that sIL-6R can act as a biologically active component of the IL-6/IL-6R system, little is known about the physiologic role of sIL-6R in vivo. Adenovirus vectors have proven to be very efficient at delivering foreign genes in vivo and we generated a recombinant adenovirus expressing biologically active rat sIL-6R and showed sustained synthesis and release of this recombinant protein, first in an in vitro system and then in an animal model.

Adenoviruses as gene transfer vectors feature several advantages, among them is the ease to manipulate their genetic material and obtain high titer virus stock, the absence of integration irito the host-cell genome, their relative safety, and their ability to infect nonreplicating cells (39). In our approach, we inserted the gene encoding the extracellular domain of rat IL-6R and its signal sequence in the $\mathrm{E} 1$ region of an El/E3 deleted adenovirus vector under the control of the human CMV (pACCMV) promoter. We first confirmed with a control virus expressing LacZ and the appropriate staining that hepatoma cells were easily infectable by adenovirus (data not shown), as others have shown $(23,24)$. RNA isolation from hepatoma cells of two different species, rat and human, demonstrate the difference of activity of the promoter, depending on the host (25). Other data from our lab indicate that the pACCMV promoter works best in human cells but is also active in rodent cells (V. Thibault et al., unpublished data). By integrating the bands on Northern and Western blots we could estimate the pACCMV promoter activity in rodent hepatoma to be around seven times less active than in human cells. In fibroblasts, the difference is even greater. Activity of the promoter is therefore highly dependent on the type of cells studied even though its activity translates into high recombinant protein production. At the peak of expression, human fibroblasts release a maximum rat sIL-6R of $13 \mu \mathrm{g} / \mathrm{ml} / 10^{5}$ cells $/ 24$ $\mathrm{hr}$, whereas rodent hepatoma cells peak at 400 $\mathrm{ng} / \mathrm{ml} / 10^{5}$ cells $/ 24 \mathrm{hr}$. This amount of protein produced is in the same range as what we and others have reported earlier with recombinant adenoviruses expressing other cytokines or proteins (26-28). The kinetics of in vitro protein production is also dependent on cell type, with steady production from hepatoma cells from Day 1 or 2 and a peak expression at Day 5 for lung fibroblasts.

Vaccination of rabbits with $10^{9}$ pfu i.p. of adenovirus vector expressing rat sIL-6R and several boosts with baculovirus-purified recombinant material generated specific antibodies against rat sIL-6R. An ELISA was established on the basis of an antibody capture assay. The range of linearity was between 10 to $500 \mathrm{ng} / \mathrm{ml}$. We could not detect (by ELISA or Western blot) sIL-6R in any of the control virus-infected cells or BAL from control virus-infected animals. Recombinant rat IL-6 did not interact with the antibody. To our knowledge, this is the first ELISA to measure rat sIL-6R.

We and others have previously characterized the distribution of adenovirus-mediated gene delivery to the lungs $(21,29,30,40,41)$. The primary cell infected is the bronchial epithelial cell, but all major cell types within the airways are infectable, and access to the lung space allows assessment of the production kinetics of the transgene through bronchoalveolar lavage. Previous experiments in our lab have shown transient expression of the transgene for at least 5 days and up to 


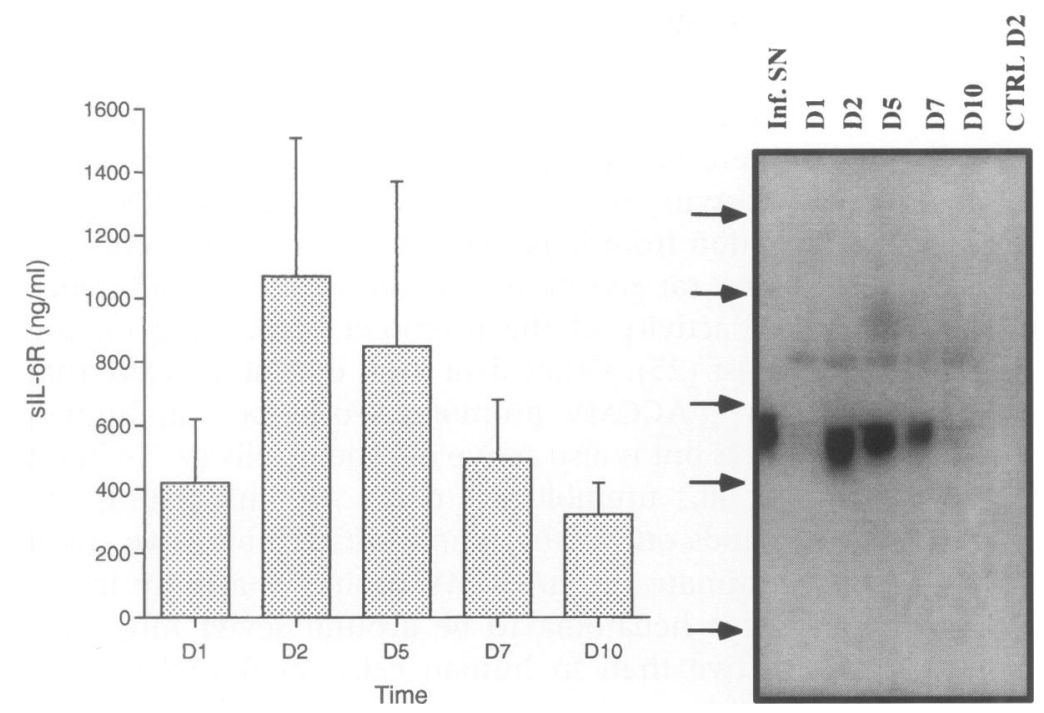

FIG. 6. Rat sIL-6R protein expression in the lung

Rats were injected intratracheally with $10^{9}$ pfu of Ad5.rsIL-6R and BAL were performed at different time points (Day 1 to Day 10). Rat sIL-6R was measured by ELISA as described. Results are expressed as mean \pm SEM. BAL fluid was subject to Western blot analysis as described in Fig. 3.
15 days in this strain (Sprague-Dawley) of rats $(21,40,41)$. Others have mostly used Cotton-rats, making comparison difficult (31-33). Rat sIL-6R production in vector-infected rats started at Day 1 and reached its maximum by Day 2 , and we could still detect raised levels of the protein to Day 10 after infection. The protein present in BAL fluid had the same characteristics as rsIL-6R produced in vitro. The in vivo expression kinetics resemble those seen in hepatoma cells, and at the peak (Day 2) we measured a mean of $1100 \mathrm{ng} /$ $\mathrm{ml}$, decreasing by Day 10 to $400 \mathrm{ng} / \mathrm{ml}$ BAL fluid, levels which are significantly above those detected for human sIL-6R (up to $0.7 \mathrm{ng} / \mathrm{ml}$ ) in human BAL fluid, of patients with or without interstitial lung disease (44). Total lung sIL-6R in the human is approximately $50 \mathrm{ng} / \mathrm{lung}$, whereas the gene transfer system used here generated levels from $1.2 \mu \mathrm{g} / \mathrm{lung}$ to $3.3 \mu \mathrm{g} / \mathrm{lung}$ in the rat. Even at the latest time point examined postinfection (Day 10), the levels of rat sIL-6R were still above those reported in mouse serum and certainly high enough to generate biological activity. In vitro experiments showed a drop in expression level after Day 5 in fibroblasts and a sustained transgene production in hepatocytes up to Day 8. In vivo, the expression kinetics are different and the influence of the host immune response against the viral vector (not against the homologous rat transgene) is clearly demonstrated in animal models and seems to shorten the period of expression (34-36). Rat sIL-6R produced locally might render IL-6R-negative cells sensitive to the action of IL- 6 and therefore could increase the intensity of the immune response.
We did not measure IL- 6 in these animals, as we have previously shown that the presence of rat sIL-6R in samples markedly affects the B9 hybridoma cell activity assay for rat IL-6 (42). We currently do not possess an ELISA for rat IL-6, but this will be needed to determine the effect of receptor overexpression on IL-6 levels.

We have previously shown that purified recombinant rat sIL-6R could confer responsiveness of human hepatoma cells (HepG2) to rat IL-6, this system being the most convenient to test for rat sIL-6R activity (42). Ad5.rsIL-6R infection of HepG2 cells did not modify basal or human IL-6-stimulated acute phase protein production, but stimulation of Ad5.rsIL-6R-infected HepG2 with rat IL-6 induced a strong acute phase protein response not seen in cells infected with control virus (Ad5.E1.LacZ). We were able to induce a 3 -fold increase in $\alpha$-1 antichymotrypsin production, a level of stimulation identical to the one induced by human IL- 6 on HepG 2 cells. The intensity of the response seen after infection with rat sIL-6R producing vector is very similar to that seen using a combination of recombinant rat sIL-6R and rat IL-6 (42). This experiment demonstrates the potency of thc approach to provide a receptor component to a cell that lacks part of one receptor. This system may represent, to a certain extent, what happens in vivo, where some cells may shed or release sIL-6R and thereby provide IL-6R-negative cells with the appropriate receptor for IL-6 stimulation.

Adenoviral vectors exhibit numerous advantages for looking for biologic activities of proteins 
in vivo or in vitro. In most cases, they induce only minimal inflammatory changes and a transient but sustained (up to 10 days) production of the transgene of interest (38). In our case, we demonstrated for the first time adenovirus-mediated production and bioactivity of rat sIL-6R in an in vivo model. This gene transfer approach coupled with an immunoassay and bioassay for sIL-6R in the rat will facilitate future detailed investigations into the physiologic and pathologic roles of the soluble IL-6 receptor.

\section{ACKNOWLEDGMENTS}

The excellent work of Jane Ann Schroeder, Xueya Feng, and Duncan Chong is gratefully acknowledged. This work was supported by MRC Canada and NCI Canada. F.L.G. is a Terry Fox Scientist of NCIC. V.T. is supported by a scholarship from Rhone Poulenc Rorer.

\section{REFERENCES}

1. Hirano T, Taga T, Matsuda T, et al. (1990) Interleukin 6 and its receptor in the immune response and hematopoiesis. Int. J. Cell Cloning 8(Suppl 1): 155-66 [discussion 166167].

2. Baumann H, Gauldie J. (1994) The acute phase response [Review]. Immunol. Today 15: 74-80.

3. Ward LD, Howlett GJ, Discolo G, et al. (1994) High affinity interleukin-6 receptor is a hexameric complex consisting of two molecules each of interleukin-6, interleukin-6 receptor, and gp-130. J. Biol. Chem. 269: 23286-23289.

4. Kishimoto T, Akira S, Narazaki M, Taga T. (1995) Interleukin-6 family of cytokines and gp130 [Review]. Blood 86: 1243-1254.

5. Taga T, Hibi $M$, Hirata $Y$, et al. (1989) Interleukin-6 triggers the association of its receptor with a possible signal transducer, gp130. Cell 58: 573-581.

6. Florillo MT, Toniatti C, Van Snick J, Ciliberto G. (1992) Expression of the murine interleukin 6 receptor in hepatoma cells: The intracytoplasmic domain is not required for interleukin 6 signal transduction. Eur. J. Immunol. 22: 799-804.

7. Hooper WC, Phillips DJ, Ribeiro M, Benson J, Evatt BL. (1995) IL-6 upregulates protein
S expression in the HepG-2 hepatoma cells. Thromb. Haemost. 73: 819-824.

8. Mihara M, Moriya Y, Kishimoto T, Ohsugi Y. (1995) Interleukin-6 (IL-6) induces the proliferation of synovial fibroblastic cells in the presence of soluble IL-6 receptor. Br. J. Rheumatol. 34: 321-325.

9. Mackiewicz A, Schooltink H, Heinrich PC, Rose-John S. (1992) Complex of soluble human IL-6-receptor/IL-6 up-regulates expression of acute-phase proteins. J. Immunol. 149: 2021-2027.

10. Mackiewicz A, Rose-John S, Schooltink H, Laclak M, Gorny A, Heinrich PC. (1992) Soluble human interleukin-6-receptor modulates interleukin-6-dependent $\mathrm{N}$-glycosylation of alpha 1-protease inhibitor secreted by HepG2 cells. FEBS Lett. 306: 257-261.

11. Tamura T, Udagawa N, Takahashi $\mathrm{N}$, et al. (1993) Soluble interleukin-6 receptor triggers osteoclast formation by interleukin 6 . Proc. Natl. Acad. Sci. U.S.A. 90: 11924-1 1928.

12. Schobitz B, Pezeshki G, Pohl T, et al. (1995) Soluble interleukin-6 (IL-6) receptor augments central effects of IL-6 in vivo. FASEB J. 9: 659-664.

13. Igleslas $M$, Plowman GD, Woodworth $C D$. (1995) Interleukin 6 and interleukin-6-soluble receptor regulate proliferation of normal, human papillomavirus-immortalized, and carcinoma-derived cervical cells in vitro. Am. J. Pathol. 146: 944-952.

14. De Benedetti F, Massa $M$, Pignatti $P$, Albani S, Novick D, Martini A. (1994) Serum soluble interleukin 6 (IL-6) receptor and IL-6/ soluble IL-6 receptor complex in systemic juvenile rheumatoid arthritis. J. Clin. Invest. 93: 2114-2119.

15. Gaillard JP, Bataille R, Brailly H, et al. (1993) Increased and highly stable levels of functional soluble interleukin-6 receptor in sera of patients with monoclonal gammopathy. Eur. J. Immunol. 23: 820-824.

16. Honda $M$, Yamamoto $S$, Cheng $M$, et al. (1992) Human soluble IL-6 receptor: its detection and enhanced release by HIV infection. J. Immunol. 148: 2175-2180.

17. Frieling JT, van Deuren $M$, Wijdenes J, et al. (1995) Circulating interleukin-6 receptor in patients with sepsis syndrome. J. Infect. Dis. 171: 469-472.

18. Berkner KL. (1988) Development of adenovirus vectors for the expression of heterologous genes. BioTechniques 6: 616-629.

19. Chomczynski P, Sacchl N. (1987) Single-step 
method of RNA isolation by acid guanidinium thiocyanate-phenol-chloroform extraction. Anal. Biochem. 162: 156.

20. Baumann M, Baumann H, Fey GH. (1990) Molecular cloning, characterization and functional expression of the rat liver interleukin 6 receptor. J. Biol. Chem. 265: 1985319862.

21. Xing Z, Braciak T, Jordana M, Croitoru K, Graham FL, Gauldie J. (1994) Adenovirusmediated cytokine gene transfer at tissue sites. Overexpression of IL-6 induces lymphocytic hyperplasia in the lung. J. Immunol. 153: 4059-4069.

22. Graham FL, Prevec L. (1991) Manipulation of adenovirus vectors. In: Murray EJ (ed). Methods in Molecular Biology, Vol. 7. Humana Press, Clifton, NJ, pp. 109-128.

23. Jaffe HA, Danel C, Longenecker G, et al. (1992) Adenovirus-mediated in vivo gene transfer and expression in normal rat liver. Nat. Genet. 1: 372-378.

24. Yang Y, Raper SE, Cohn JA, Engelhardt JF, Wilson JM. (1993) An approach for treating the hepatobiliary disease of cystic fibrosis by somatic gene transfer. Proc. Natl. Acad. Sci. U.S.A. 90: 4601-4605.

25. Xu ZZ, Krougliak V, Prevec L, Graham FL, Both GW. (1995) Investigation of promoter function in human and animal cells infected with human recombinant adenovirus expressing rotavirus antigen VP7sc. J. Gen. Virol. 76: 1971-1980.

26. Braciak TA, Mittal SK, Graham FL, Richards CD, Gauldie J. (1993) Construction of recombinant human type 5 adenoviruses expressing rodent IL- 6 genes. An approach to investigate in vivo cytokine function. $\mathrm{J}$. Immunol. 151: 5145-5153.

27. Lindley $\mathrm{T}$, Virk KP, Ronchetti-Blume $M$, et al. (1994) Construction and characterization of adenovirus co-expressing hepatitis B virus surface antigen and interleukin-6. Gene 138: 165-170.

28. Kopfler WP, Willard M, Betz T, Willard JE, Gerard RD, Meidell RS. (1994) Adenovirusmediated transfer of a gene encoding human apolipoprotein A-I into normal mice increases circulating high-density lipoprotein cholesterol. Circulation 90: 1319-1327.

29. Crystal RG, McEIvaney NG, Rosenfeld MA, et al. (1994) Administration of an adenovirus containing the human CFTR CDNA to the respiratory tract of individuals with cystic fibrosis. Nat. Genet. 8: 42-51.
30. Rosenfeld MA, Ronald G, Crystal RG. (1993) Gene therapy for pulmonary diseases [Review]. Pathol. Biol. 41: 677-680.

31. Rosenfeld MA, Siegfried W, Yoshimura K, et al. (1991) Adenovirus mediated transfer of a recombinant alpha 1-antitrypsin gene to the lung epithelium in vivo. Science 252: 431434.

32. Rosenfeld MA, Yoshimura K, Trapnell BC, et al. (1992) In vivo transfer of the human cystic fibrosis transmembrane conductance regulator gene to the airway epithelium. Cell 68: 143-155.

33. Zabner J, Petersen DM, Puga AP, et al. (1994) Safety and efficacy of repetitive adenovirus-mediated transfer of CFTR CDNA to airway epithelia of primates and cotton rats. Nat. Genet. 6: 75-83.

34. Yang Y, Li Q, Ertl HC, Wilson JM. (1995) Cellular and humoral immune responses to viral antigens create barriers to lung-directed gene therapy with recombinant adenoviruses. J. Virol. 69: 2004-2015.

35. Yang Y, Wilson JM. (1995) Clearance of adenovirus-infected hepatocytes by MHC class I-restricted CD4 ${ }^{+}$CTLs in vivo. J. Immunol. 155: 2564-2570.

36. Engelhardt JF, Litzky L, Wilson JM. (1994) Prolonged transgene expression in cotton rat lung with recombinant adenoviruses defective in E2a. Hum. Gene Ther. 5: 1217-1229.

37. Baumann H, Richards C, Gauldie J. (1987) Interaction among hepatocyte-stimulating factors, interleukin-1, and glucocorticoids for regulation of acute phase plasma proteins in human hepatoma (HepG2) cells. J. Immunol. 139: 4122-4128.

38. Brody SL, Crystal RG. (1994) Adenovirusmediated in vivo gene transfer [Review]. Ann. N. Y. Acad. Sci. 716: 90-101.

39. Bramson JL, Graham FL, Gauldie J. (1995) The use of adenoviral vectors for gene therapy and gene transfer in vivo. Curr. Opin. Biotechnol. 6: 590-595.

40. Braciak TA, Bacon K, Xing Z, et al. (1996) Overexpression of RANTES using a recombinant adenovirus vector induces the tissue directed recruitment of monocytes to the lung. J. Immunol. 157: 5076-5084.

41. Foley R, Driscoll KE, Wan YH, et al. (1996) Adenoviral gene transfer of macrophage inflammatory protein-2 in rat lung. Am. J. Pathol. 149: 1395-1403.

42. Thibault V, Terlain B, Gauldie J. (1996) Characterization and biological activities of 
recombinant rat soluble interleukin-6 receptor. J. Interferon Cytokine Res. 16: 973-981.

43. Frieling JT, Sauerwin RW, Wijdenes J, Hendriks T, van der Linden CJ. (1994) Soluble interleukin 6 receptor in biological fluids from human origin. Cytokine 6: 376-381.

44. Yokoyama A, Kohno N, Hirasawa Y, et al. (1995) Evaluation of soluble IL-6 receptor concentration in serum and epithelial lining fluid from patients with interstitial lung diseases. Clin. Exp. Immunol. 100: 325-329.

45. Kollis J, Peppl K, Silva M, Beutler B. (1994) Prolonged and effective blockade of tumor necrosis factor activity through adenovirusmediated gene transfer. Proc. Natl. Acad. Sci. U.S.A. 91: 215-219.

Communicated by A. Cerami. Accepted May 8, 1997. 